\title{
Finding the Most Metal-poor Stars of the Galactic Halo with the Hamburg/ESO Objective-prism Survey
}

\author{
Norbert Christlieb \\ Hamburger Sternwarte \\ Gojenbergsweg 112 \\ D-21029 Hamburg \\ Germany \\ E-Mail: nchristlieb@hs.uni-hamburg.de \\ URL: http://www.hs.uni-hamburg.de/DE/Ins/Per/Christlieb/
}

\begin{abstract}
I review the status of the search for extremely metal-poor halo stars with the Hamburg/ESO objective-prism survey (HES). 2194 candidate metal-poor turnoff stars and 6133 giants in the magnitude range $14 \lesssim B \lesssim 17.5$ have been selected from 329 (out of 380) HES fields, covering an effective area of 6400 square degrees in the southern extragalactic sky. Moderate-resolution followup observations for 3200 candidates have been obtained so far, and $\sim 200$ new stars with $[\mathrm{Fe} / \mathrm{H}]<-3.0$ have been found, which trebles the total number of such extremely low-metallicity stars identified by all previous surveys.

We use VLT-UT2/UVES, Keck/HIRES, Subaru/HDS, TNG/SARG, and Magellan/MIKE for high-resolution spectroscopy of HES metal-poor stars. I provide an overview of the scientific aims of these programs, and highlight several recent results.
\end{abstract}

\section{Introduction}

Extremely metal-poor (EMP) stars, defined here as stars with $1 / 1000$ th of the metal content of the Sun (i. e., $[\mathrm{Fe} / \mathrm{H}]<-3.0)^{1}$, permit astronomers to study the earliest epochs of Galactic chemical evolution, since the atmospheres of these old, low-mass stars retain, to a large extent, detailed information on the chemical composition of the interstellar medium (ISM) at the time and place of their birth. These stars are hence the local equivalent of the high redshift Universe (for recent reviews on EMP stars see Cayrel 1996, Beers 1999, 2000; Preston 2000).

\footnotetext{
${ }^{1}[\mathrm{~A} / \mathrm{B}]=\log _{10}\left(N_{\mathrm{A}} / N_{\mathrm{B}}\right)-\log _{10}\left(N_{\mathrm{A}} / N_{\mathrm{B}}\right)_{\odot}$, for elements $\mathrm{A}$ and $\mathrm{B}$.
} 
In the past decade, numerous studies have focused on abundance analysis of very metal-poor stars. In the early works of McWilliam et al. (1995b) and Ryan et al. (1996), it was found that many elements show a sudden change in the slope of their abundances relative to $\mathrm{Fe}$, near $[\mathrm{Fe} / \mathrm{H}]=-2.5$. Other elements, including $\mathrm{Ba}$ and $\mathrm{Sr}$, show a large scatter from star-to-star at very low $[\mathrm{Fe} / \mathrm{H}]$, indicating that the gas clouds from which these stars formed were polluted by the products of only a few, if not single, supernovae (see, e. g., Shigeyama \& Tsujimoto 1998; Tsujimoto et al. 2000). Thus, with individual EMP stars we can indirectly study the first generation of massive stars, which exploded in supernovae of type II (hereafter SN II). Furthermore, Karlsson \& Gustafsson (2001) have shown that the inspection of patterns seen in abundance correlation diagrams of a large, homogeneously-analyzed sample of stars allows one to constrain theoretical yield calculations, and to perhaps even determine the mass function of the first generation of stars.

EMP stars also contribute to observational cosmology: The abundances of light elements (in particular Li), through models of Big Bang nucleosynthesis, put limits on the baryonic content of the Universe (e. g., Ryan et al. 1999), and individual age determinations of the oldest stars - either by nucleo-chronometry (e. g., Cowan et al. 1999; Cayrel et al. 2001), or by means of application of stellar evolutionary tracks (e. g., Fuhrmann 1998) - yield a lower limit for the age of the Galaxy, and hence the Universe.

However, it should be recognized that, owing to their rarity, the road to obtaining elemental abundances for EMP stars is long and arduous. The process involves three major observational steps (see Figure 1): (1) a wide-angle survey must be carried out, and candidates for metal-poor stars have to be selected; (2) moderate-resolution ( $\sim 2 \AA$ ) spectroscopic follow-up observations of the candidates needs to be done in order to validate the genuinely metal-poor stars among them; and (3) high-resolution spectroscopy has to be obtained. Step (1) can be carried in many different ways (for a review, see Beers 2000), for example, with proper-motion surveys or photometric surveys. However, spectroscopic surveys are the most efficient way to find large numbers of EMP stars, and they have the advantage that the samples they yield are not kinematically biased, as opposed to proper-motion selected samples.

Until recently, the main source of EMP stars was the HK survey of Beers and colleagues (Beers et al. 1985; Beers 1999), which so far has yielded about 100 stars with $[\mathrm{Fe} / \mathrm{H}]<-3.0$. For most of them, high resolution $(R>40000)$ spectroscopy has now been obtained (see, e. g., Ryan et al. 1996; Norris et al. 2001). As a consequence, the abundance trends of many elements are now well-determined down to metalicities of $[\mathrm{Fe} / \mathrm{H}] \sim-3.5$.

Still larger samples of EMP stars are needed to identify the small subset of stars that are even more exotic then the EMP stars themselves, e. g., stars with strong enhancements of the r-process elements, suitable for nucleo-chronometric age dating (Cowan et al. 1997, 1999; Cayrel et al. 2001; Hill et al. 2002). The frequency of these stars among metal-poor giants with $[\mathrm{Fe} / \mathrm{H}]<-2.5$ has been found in the HK survey to be as low as $\sim 3 \%$, a paucity confirmed by recent HES results (see Section 4.2 for details). HE $0107-5240$ is another example of an extremely rare star. It is a giant with $[\mathrm{Fe} / \mathrm{H}]=-5.3$ (Christlieb et al. 2002a), which was found during follow-up observations of $\sim 200$ stars with $[\mathrm{Fe} / \mathrm{H}]<-3.0$ (see Section 4.4 below). 


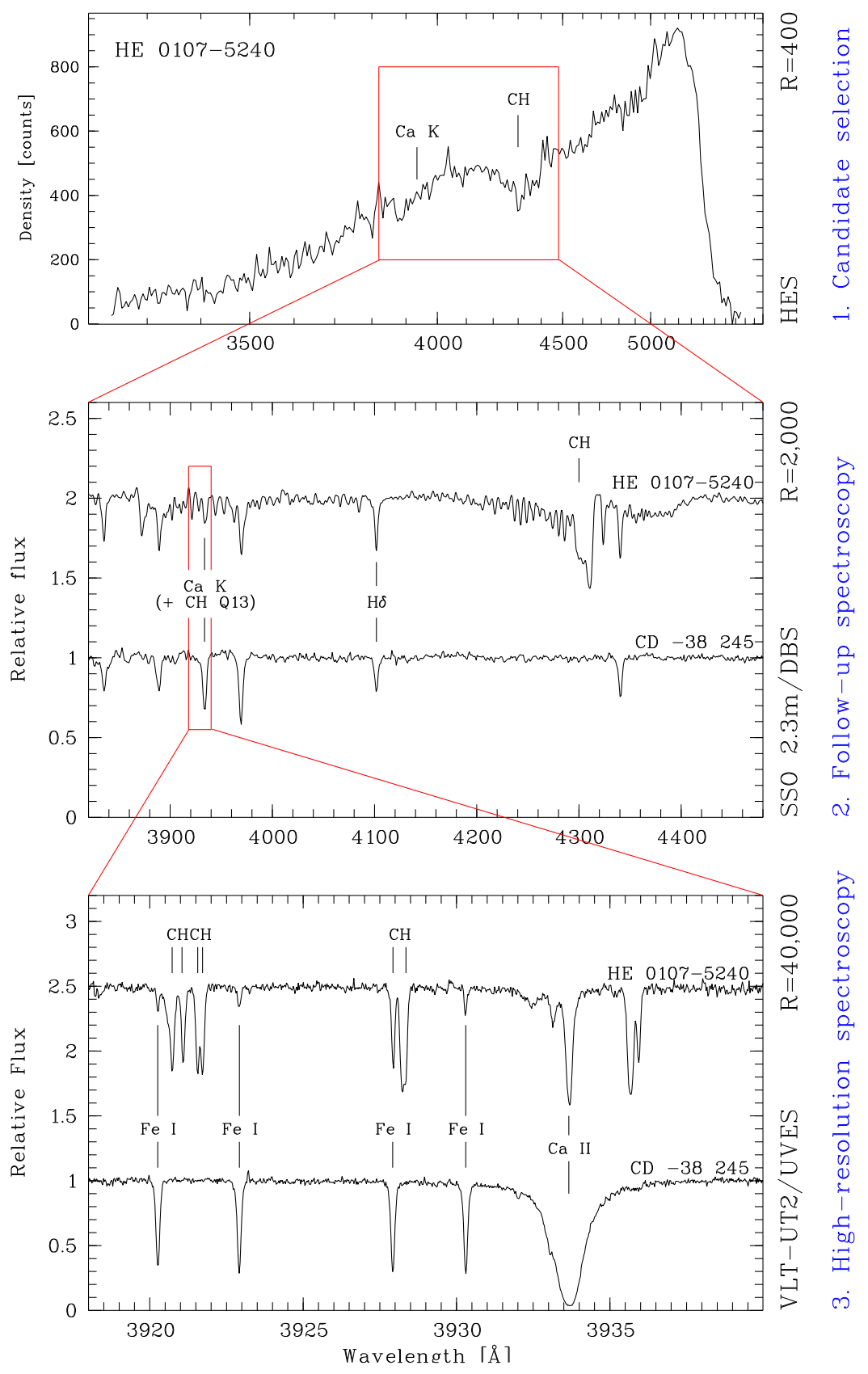

Figure 1: The three major observational steps towards obtaining elemental abundances of extremely metal-poor stars: (1) objective-prism spectra, yielding candidate metal-poor stars; (2) vetting of candidates by moderate-resolution follow-up spectroscopy; (3) high-resolution spectroscopy of confirmed candidates. 
Furthermore, stars of the lowest metallicity place strong constraints on the nature of the Metallicity Distribution Function (MDF), which presumably arises from the ejected yields of the first generations of stars. For example, with the exception of the star at $[\mathrm{Fe} / \mathrm{H}]=-5.3$, it appears that the lowest abundance stars in the Galaxy reach no lower than $[\mathrm{Fe} / \mathrm{H}] \sim-4.0$, hence this is the level of early "pollution" of the ISM one might demand to be reached by the products of first generation stars. One also would like to test whether the MDF, as reflected by observations of halo stars in the Solar neighborhood, remains constant or varies with Galactocentric distance.

The Hamburg/ESO objective-prism survey (HES; Wisotzki et al. 2000) offers the opportunity to increase the number of EMP stars by at least a factor of five with respect to the HK survey, since the HES is more than 1.5 mag deeper, and covers regions of the sky not included in the HK survey (see Figure 2). For a detailed comparison of the HES with the HK survey, see Christlieb \& Beers (2000).
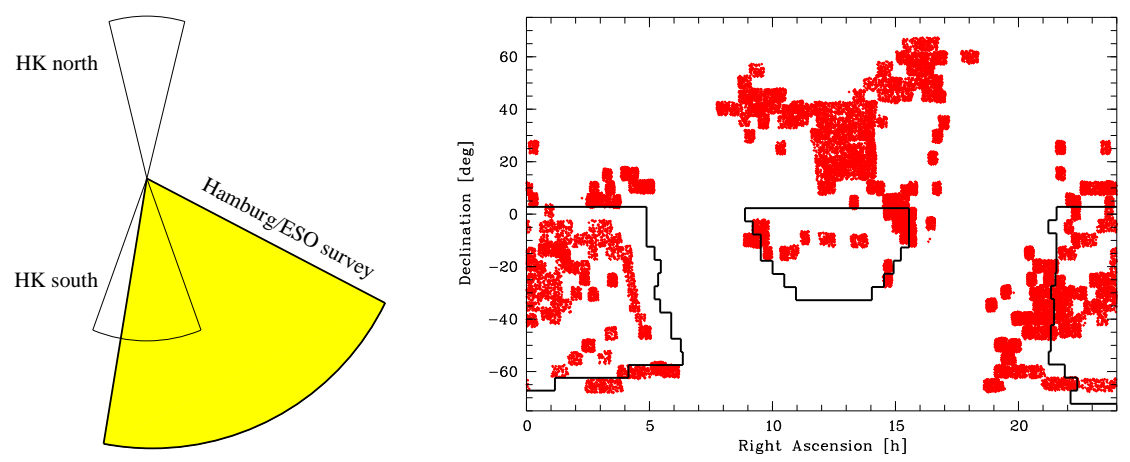

Figure 2: Comparison of the survey volumes of the HK and HE surveys (left panel); comparison of HES area (framed) with HK survey area (right panel). Grey areas denote HK survey plates.

The HES covers the total southern $\left(\delta<-2.5^{\circ}\right)$ extragalactic $\left(|b| \gtrsim 30^{\circ}\right)$ sky. The HES objective-prism plates were obtained with the ESO $1 \mathrm{~m}$-Schmidt telescope, and scanned and reduced at Hamburger Sternwarte. The faintest HES metal-poor stars have $B \sim 17.5 \mathrm{mag}$; stars this faint are observable at high spectral resolution using currently existing telescope/instrument combinations.

\section{Selection of metal-poor candidates}

Metal-poor turnoff stars are selected in the HES database of digital objective-prism spectra (for examples see Fig. 2) by automatic spectral classification (Christlieb et al. 2002b). Cooler stars $(B-V>0.5)$ are currently selected by applying an empirical cutoff line in $(B-V)$ versus KP space, where KP is the Ca II K line index defined by Beers et al. 1999). 
The selection has so far been restricted to unsaturated point sources. For stars brighter than $B \sim 14 \mathrm{mag}$, saturation effects (in particular in the red portions of the prism spectra) begin to occur. Investigations on methods for recovering at least a portion of these brighter candidates are underway (Frebel et al., in preparation). The importance of the bright sources comes from the relative ease with which highresolution spectroscopy can be obtained. Extended sources are being rejected because spatial extension leads to smeared-out spectral lines in slitless spectra, and therefore many galaxies would otherwise enter the candidate sample as false positives.

A total of 2194 candidate metal-poor turnoff stars have been selected from the 329 (out of 380) HES fields that are currently used for stellar work. These fields cover a nominal area of $8225 \mathrm{deg}^{2}$ of the southern extragalactic sky. The covered area is reduced to an effective area of $6400 \mathrm{deg}^{2}$ due to overlapping spectra (Wisotzki et al. 2000; Christlieb et al. 2001a). In addition to the turnoff stars, 6133 metal-poor candidates with $0.5<B-V<1.2$ were selected, including 1785 (i.e., $\sim 30 \%$ ) candidates expected to have large over-abundances of carbon $([\mathrm{C} / \mathrm{Fe}]>1.0)$. These stars are readily identifiable in the HES from their strong $\mathrm{CH} \mathrm{G}$ bands (see Figure 4). A sample of 403 faint high latitude carbon (FHLC) stars has already been published (Christlieb et al. 2001a), and that sample contains many carbon-enhanced metal-poor (CEMP) stars as well, as recent follow-up observations have demonstrated.

As noted above, the HES is a very rich source for metal-poor giants. This is especially valuable because the present HK survey sample is dominated by hotter stars near the main-sequence turnoff, due to a temperature-related bias incurred by the initial visual selection of HK candidates. As a result, only roughly $20 \%$ of the HK stars are metal-poor giants. However, efforts are under way to recover metalpoor HK survey giants, by means of Artificial Neural Network classifications of spectra extracted from digitized HK survey plates (Rhee 2000) in combination with JHK colors from the 2MASS survey (Skrutskie et al. 1997).

\section{Follow-up observations}

Moderate resolution $(\sim 2 \AA)$ follow-up observations are needed to verify the metalpoor nature of the HES candidates. This is the bottleneck in every large survey for EMP stars, and the only way to cope with this is to establish large collaborations, involving many people, with access to many different telescopes. To provide the reader an impression of the extent of this effort, we list in Table 1 the telescopes that have been used in 2002 for follow-up of HES candidates, and the numbers of stars observed.

Follow-up spectra of $S / N>20$ at Ca II K are required to determine [Fe/H] of the metal-poor candidates with an accuracy of $0.2-0.3 \mathrm{dex}$, using the technique of Beers et al. (1999). As can be seen from Table 1, on average $\sim 20$ stars per night can be observed at a $4 \mathrm{~m}$-class telescope in single-slit mode, taking into account losses due to bad weather, moonlight, and technical downtime. Note that the candidate throughput at the $1 \mathrm{~m}$-UK Schmidt is as high achieved as by telescopes with $\sim 15$ times more collecting area, due to the availability of the 150 fiber instrument $6 \mathrm{dF}$ 

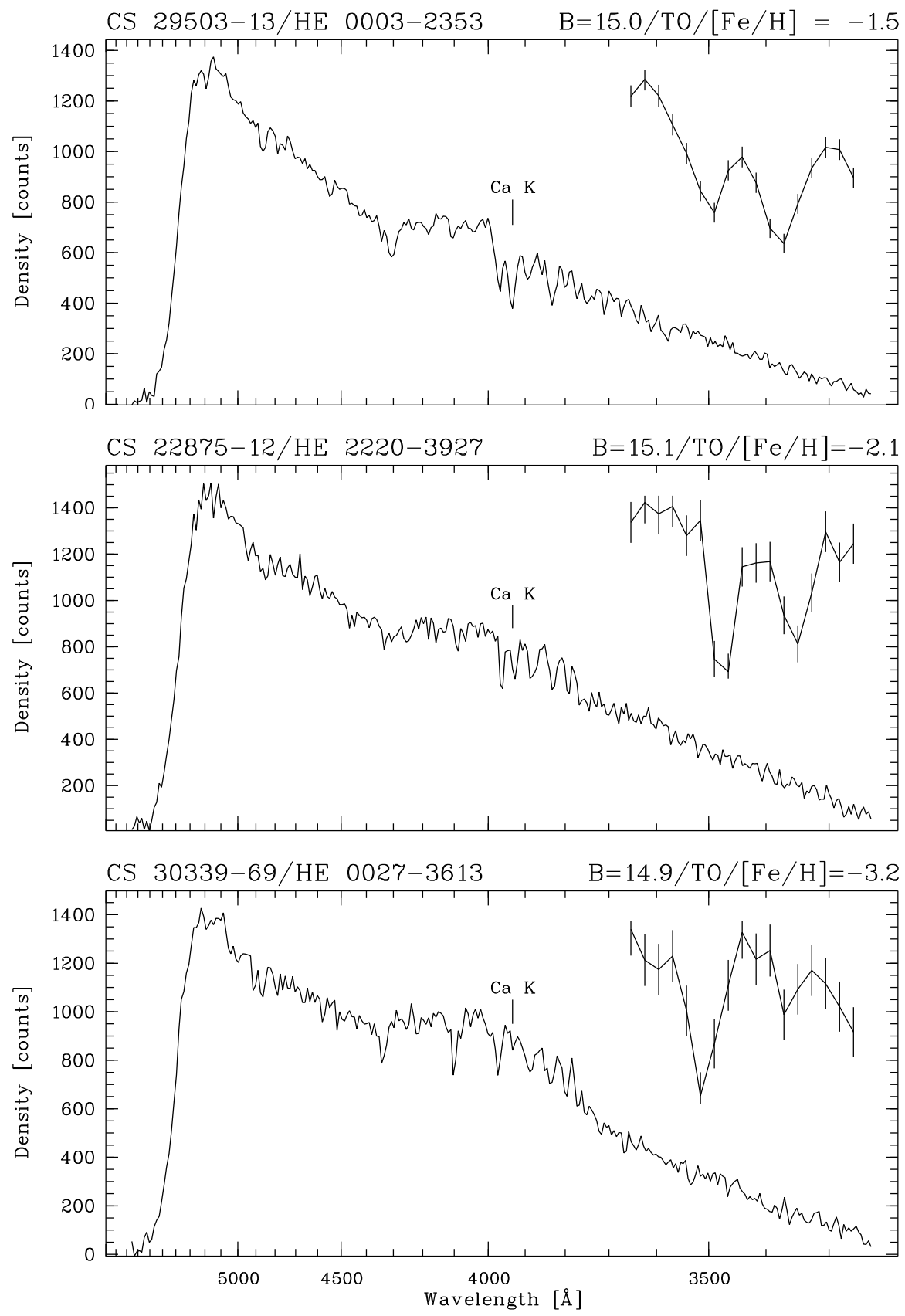

Figure 3: HES spectra of metal-poor stars found in the HK survey. Note that wavelength is decreasing from left to right. The sharp cutoff at $\sim 5400 \AA$ is due to the IIIa-J emulsion sensitivity cutoff ("red edge"). 

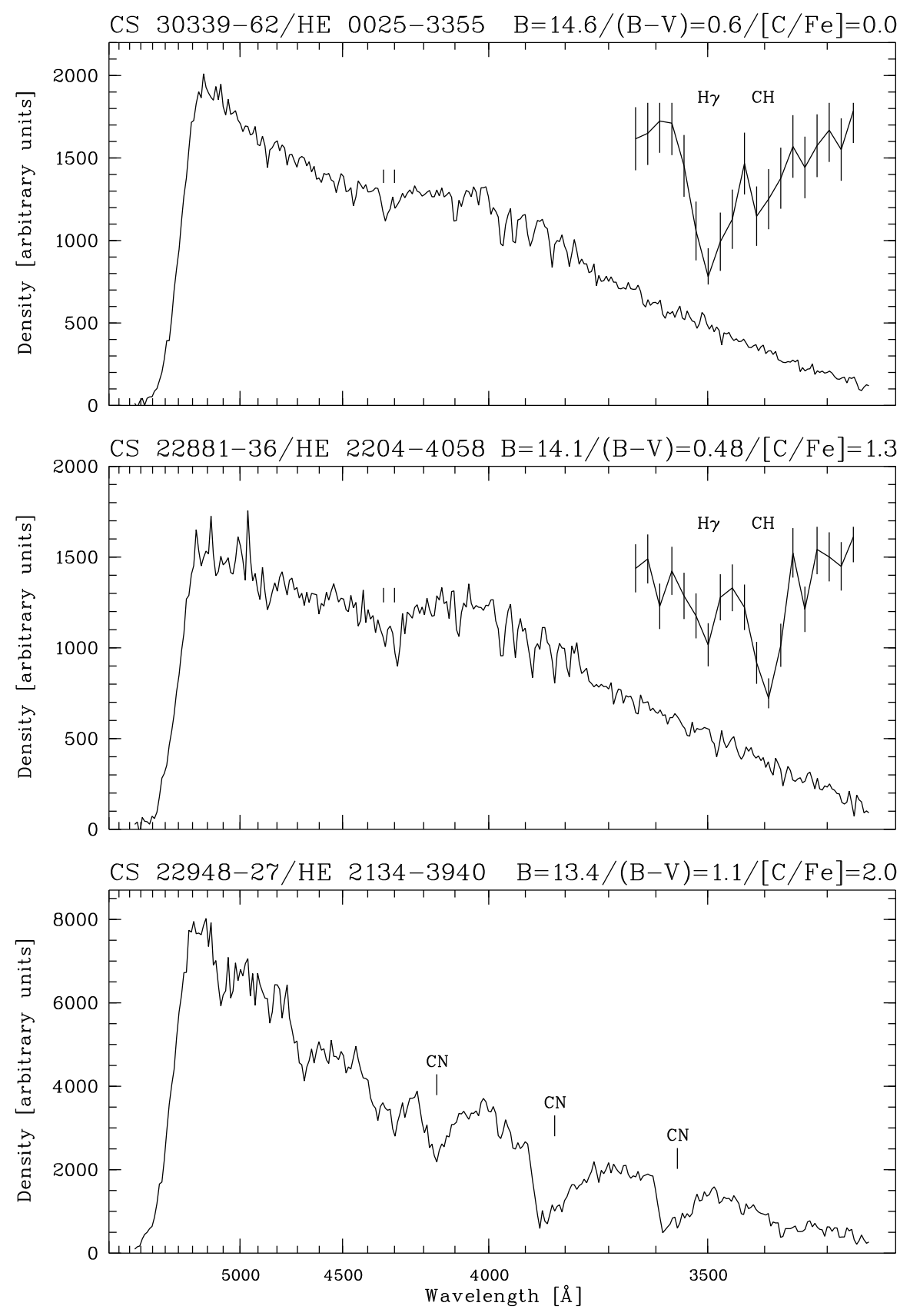

Figure 4: HES spectra of carbon-enhanced metal-poor stars from the HK survey. 
Table 1: Follow-up observations of HES metal-poor candidates in 2002.

\begin{tabular}{lrrc}
\hline \hline Telescope/Instr. & Nights & Stars & Stars/Night \\
\hline AAT/RGOS & 6 & 87 & 15 \\
ESO 3.6m/EFOSC2 & 13 & 274 & 21 \\
KPNO 4m/RCSP & 5 & 109 & 22 \\
Magellan I/B\&C & 33 & 713 & 22 \\
Palomar 200"/DS & 9 & 96 & 11 \\
SSO 2.3 m/DBS & 32 & 430 & 13 \\
UK Schmidt/6dF & 16 & 341 & 21 \\
\hline Sum/average & 114 & 2050 & 18 \\
\hline \hline
\end{tabular}

(Watson 1998). Up to three fields with 10-60 metal-poor candidates, respectively, can be observed in one night. (The remaining fibers are filled with other interesting HES stars, e. g., field horizontal-branch stars, sdB stars, and white dwarfs.) However, the faintest HES candidates $(B \gtrsim 16.5$ ) cannot be observed with UK Schmidt/6dF, since the fiber diameter is $6.7^{\prime \prime}$ on the sky (chosen to suite the 6dF Galaxy Redshift Survey), and therefore observations of point sources are sky-background limited.

As of 1 February 2003, follow-up observations for 3200 HES candidates have been obtained. Considering that an average pace of $\sim 1000$ candidates per year is the maximum one can hope to reach in the long run, and the fact that $\sim 1000$ additional, bright candidates are expected to be found in the HES, the follow-up will likely continue for another 3-5 years.

The "effective yield" of metal-poor stars in the HES, as compared to the HK survey, is presented in Table 2, and illustrated in Figure 5. The effective yield (EY) refers to the fraction of genuine stars below a given $[\mathrm{Fe} / \mathrm{H}]$ in the observed candidate sample, as defined by Beers (2000). Based on a sample of 1214 candidates, it appears that the HES is twice as efficient as the HK survey in finding stars with $[\mathrm{Fe} / \mathrm{H}]<-3.0$, when photoelectric $B V$ photometry is used in the latter survey for pre-selection, and six times as efficient compared to the HK survey without usage of $B V$ photometry. The higher yields of the HES can mainly be attributed to the fact that $B-V$ colors can be derived directly from the objective-prism spectra with an accuracy of better than $0.1 \mathrm{mag}$ (Christlieb et al. 2001b), the higher quality of the HES spectra, and the employment of quantitative selection criteria, as opposed to visual selection in the HK survey.

It should be stressed that a bias towards higher EYs may be present in the HES candidate sample investigated so far, because the best (in terms of the weakness of the Ca II K lines visible in the HES spectra) and brightest candidates have been observed first. However, when the EYs within each of the candidate classes (as assigned during the visual verification of the HES candidates) are applied to the respective fraction of candidates within the total set of HES candidates, overall EYs of $4 \%$ for stars with $[\mathrm{Fe} / \mathrm{H}]<-3.0$ result for turnoff stars and giants. If more restrictive selection criteria were to be used, the EYs could be increased to $\sim 10 \%$, but in this case $\sim 1 / 4$ of the stars with $[\mathrm{Fe} / \mathrm{H}]<-3.0$ would not be identified. 
Table 2: "Effective yields" of metal-poor stars (i. e., the fraction of genuine metalpoor stars below a given $[\mathrm{Fe} / \mathrm{H}]$ in the observed candidate sample) in the $\mathrm{HK}$ and the HE surveys. $N$ refers to the number of stars from which the statistic has been derived.

\begin{tabular}{lccrr}
\hline \hline \multirow{2}{*}{ Survey } & $N$ & \multicolumn{3}{c}{$[\mathrm{Fe} / \mathrm{H}]$} \\
& & $<-2.0$ & $<-2.5$ & $<-3.0$ \\
\hline HK survey/no $B-V$ & 2614 & $11 \%$ & $4 \%$ & $1 \%$ \\
HK survey/with $B-V$ & 2140 & $32 \%$ & $11 \%$ & $3 \%$ \\
HES (turnoff stars) & 571 & $59 \%$ & $21 \%$ & $6 \%$ \\
HES (giants) & 643 & $50 \%$ & $20 \%$ & $6 \%$ \\
\hline \hline
\end{tabular}
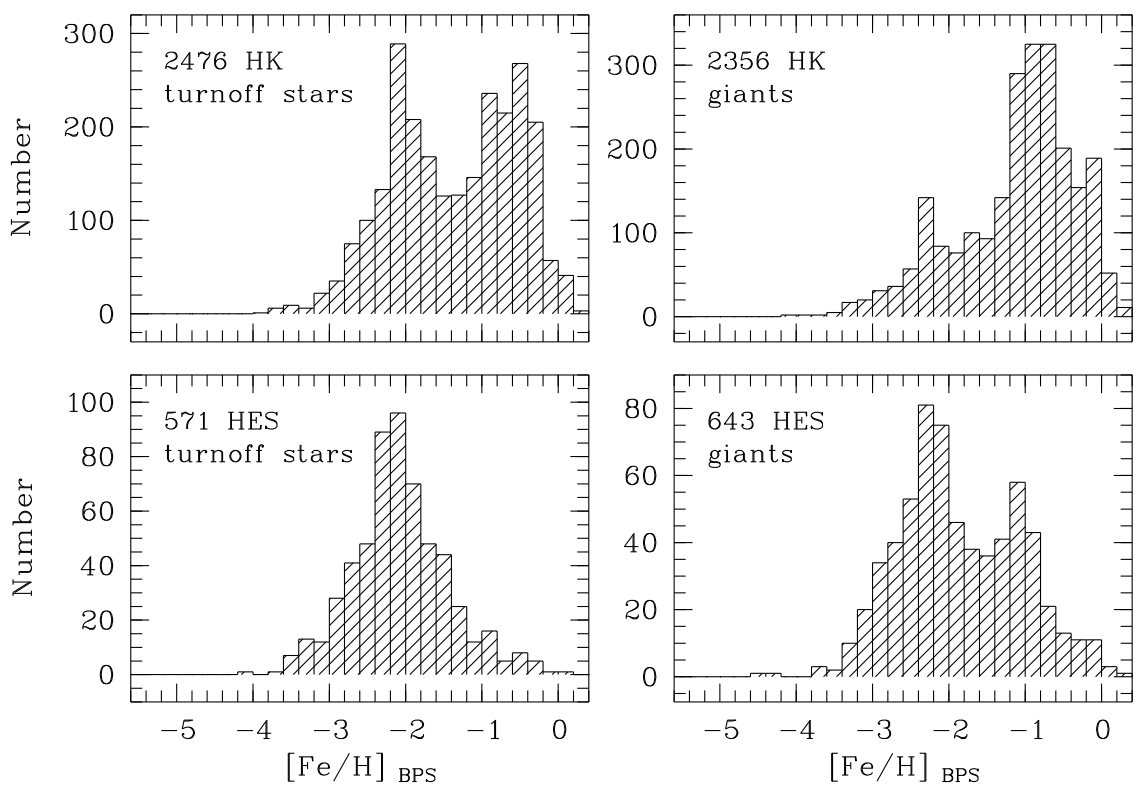

Figure 5: The observed Metallicity Distribution Functions of HES and HK turnoff stars (left panel) and giants (right panel)

Hence, a compromise is struck between the "purity" of the sample and the desire to include as many EMP stars as possible.

Using the numbers mentioned above, it can be estimated that at least 300 stars with $[\mathrm{Fe} / \mathrm{H}]<-3.0$ will eventually be found in the HES, provided that follow-up observations of all candidates are obtained (this estimate does not count the additional bright HES candidates, follow-up observations for which have only recently begun). 


\section{High-resolution spectroscopy projects}

In this section, I provide an overview of ongoing high-resolution spectroscopic analyses of confirmed metal-poor stars (mainly) from the HES, and highlight some recent results.

\subsection{The $0 Z$ (zero Z) project}

In a collaboration between Hamburger Sternwarte, Caltech, The Observatories of the Carnegie Institution of Washington (and formerly also Padua Observatory), we aim at obtaining high-resolution, high $S / N$ spectroscopy of a large sample of the most metal-poor stars, with Keck/HIRES and Magellan/MIKE. First results have been published published in Cohen et al. (2002), Carretta et al. (2002), Lucatello et al. (2003), and Cohen et al. (2003).

Highlights include the discovery of HE 0024-2523. It is a peculiar main-sequence turnoff star, and a spectroscopic binary with a period of 3.14 days. Its abundance pattern is dominated by extreme enrichment of s-process elements, likely due to mass accretion from an already evolved companion which has now probably become a white dwarf. The relative lead abundance is $[\mathrm{Pb} / \mathrm{Fe}]=+3.3 \mathrm{dex}$; the abundance ratio of $\mathrm{Pb}$ to $\mathrm{Ba}$ is even more extreme, $[\mathrm{Pb} / \mathrm{Ba}]=+1.9 \mathrm{dex}$, among the highest ever determined in a metal-poor star. The unusually short period of this $\mathrm{CH}$ star suggests a past common envelope phase.

Stars like HE 0024-2523 provide the unique opportunity to study an extinct generation of extremely metal-poor AGB stars that left their fingerprints on their less massive companions. These stars place strong observational constraints on simulations of the structure, evolution, and nucleosynthesis of AGB stars, and allow us to study the s-process at very low metalicities. Recent calculations of Goriely \& Siess (2001) predict that an efficient production of s-process elements takes place even in zero metalicity AGB stars, despite the absence of iron seeds, provided that protons are mixed into carbon-rich layers. Proton mixing results in the formation of ${ }^{13} \mathrm{C}$, which is a strong neutron source due to the reaction ${ }^{13} \mathrm{C}(\alpha, \mathrm{n}){ }^{16} \mathrm{O}$. The strong overabundances of $\mathrm{Pb}$ in HE 0024-2523 and other "Pb stars" (Aoki et al. 2000; VanEck et al. 2001; Aoki et al. 2002b) are in concert with these predictions.

Another exotic star found with Keck/HIRES observations is the EMP turnoff star HE 2148-1247. It appears that this star is enriched in both its $\mathrm{r}-$ and s-process elements, similar to CS 22898-027 (Preston \& Sneden 2001; Aoki et al. 2002b), which is almost a twin of HE 2148-1247 with respect to stellar parameters as well as abundances, and a few other stars in the literature (Preston \& Sneden 2001; Aoki et al. 2002b; Hill et al. 2000).

Cohen et al. (2003) propose a scenario for explaining the abundance pattern of HE 2148-1247. They suggest that the star is a member of a binary system (and in fact, the star shows significant radial variations) in which the former primary went through the AGB phase. In this stage, carbon and s-process elements were dumped onto the surface of HE 2148-1247 via mass transfer. The former primary then evolved into a white dwarf. Mass transfer then occurred into the reverse direction, i. e., from HE 2148-1247 to the white dwarf, which led to an accretion-induced 
collapse of the white dwarf into a neutron star. R-process nucleosynthesis then occurs in a neutrino-driven wind of the neutron star, and the nucleosynthesis products contaminate the surface layers of the star that we see today.

\subsection{The HERES project - An ESO Large Programme}

EMP stars with strong enhancements of r-process elements, like CS 22892-052 (McWilliam et al. 1995; Sneden et al. 1996; Cowan et al. 1997), and CS 31082-001 (Cayrel et al. 2001; Hill et al. 2002), are suitable for individual age determinations through the detection of the long-lived radioactive species thorium and/or uranium. Such old stars can therefore provide lower limits to the age of the Galaxy, and hence the Universe.

However, it appears that the pairs of elements used as chronometers need to be chosen very carefully. The results of Hill et al. (2002) suggest that comparison of the abundance of Th with that of the stable r-process element Eu does not yield reliable ages for all stars, and in particular an unrealistically small age for CS 31082001. $\mathrm{Th} / \mathrm{Eu}$ is roughly a factor of three higher in CS 31081-001 than observed in CS 22892-052, yielding a radioactive decay age for CS 31082-001 that is younger than the Sun! U/Th is expected to be a more accurate chronometer, due to the fact that ${ }^{238} \mathrm{U}$, with a half-life as "short" as $4.5 \mathrm{Gyr}$ (for comparison, ${ }^{232} \mathrm{Th}$ has a halflife of $14 \mathrm{Gyr}$ ), is involved. Furthermore, recent theoretical studies on r-process nucleosynthesis (Wanajo et al. 2002; Schatz et al. 2002) reveal that this chronometer is less sensitive to uncertainties of nuclear physics than any other chronometer that is amenable to observation in EMP stars.

Unfortunately, it is observationally very challenging to detect uranium in metalpoor stars; CS 31081-001 was the first one for which such a detection has been made. This is because the strongest spectral line in the optical, U II $3859.57 \AA$, is very weak (see Figure 9 of Hill et al. 2002), even if the enhancement of r-process elements is as high as $[\mathrm{r} / \mathrm{Fe}]=1.7 \mathrm{dex}$, as observed in CS 31081-001. Therefore, spectra of $S / N>200$ per pixel and $R \geq 75000$ are required for derivation of accurate $\mathrm{U}$ abundances. Also, one has to contend with the difficulty that, if the carbon abundance is significantly enhanced with respect to iron, as is the case for CS 22892-052 $([\mathrm{C} / \mathrm{Fe}]=0.98$; McWilliam et al. 1995), the $\mathrm{U}$ line is blended with a $\mathrm{CN}$ line. As a result, measurement of an $\mathrm{U}$ abundance is impossible for CS 22892-052.

Quite apart from their obvious use in individual age determination, strongly $\mathrm{r}$ process-enhanced metal-poor stars allow one to study r-process nucleosynthesis in general, and possibly to identify the site(s) of the r-process. While the abundance pattern of CS 22892-052 for the heavier stable neutron-capture elements ( $Z \geq 56)$, and the abundances of the elements in the range $56 \leq \mathrm{Z} \leq 72$ in CS 31082-001 match a scaled solar r-process pattern very well, deviations are seen for some of the lighter neutron-capture elements in both stars, and for Th (as discussed above) and possibly Os in CS 31082-001. It has therefore been suggested (Sneden et al. 2000; Hill et al. 2002) that (at least) two r-processes must be at work.

Given these results, it is clear that a much larger sample of strongly r-processenhanced metal-poor stars is required. The Hamburg/ESO $R$-processed Enhanced 
star $S$ urvey is aiming to increase the number of strongly r-process-enhanced stars to (conservatively) $\sim 5-10$, and to study a major fraction of these by means of high-resolution, high signal-to-noise UVES spectra. The project is carried out in the framework of an ESO Large Programme (P. I. Christlieb), which was recently approved for an initial period of one year.

Stars with strong r-process enhancement can be readily recognized from their prominent Europium lines. For example, CS 22892-052 has an Eu II $4129.7 \AA$ line as strong as $132 \mathrm{~m} \AA$. This means that r-process-enhanced stars can be identified from "snapshot" high-resolution spectra, i.e., $R=20000$ and $S / N=30$. Such spectra can easily be obtained, e. g., with VLT-UT2/UVES, or with Subaru/HDS, in $20 \mathrm{~min}$ (including overheads) for a $B=15 \mathrm{mag}$ star, even under unfavorable conditions (e. g., full moon, seeing $2^{\prime \prime}$, thin clouds). About half of our follow-up efforts presented above are seeking confirmation of giants with $[\mathrm{Fe} / \mathrm{H}] \leq-2.5$ for this project.

To date, a total of 152 targets, 128 of them from the HES, have been observed in snapshot mode with UVES. Among the 98 stars with $[\mathrm{Fe} / \mathrm{H}]<-2.5$ dex, four with $[\mathrm{r} / \mathrm{Fe}]>+1.0$ dex have been found (three of them are HES stars). Among the 54 stars with $[\mathrm{Fe} / \mathrm{H}] \geq-2.5 \mathrm{dex}$, one with $[\mathrm{r} / \mathrm{Fe}]>+1.0 \mathrm{dex}$ has been found. Higher resolution and $S / N$ spectra of most of these stars is currently being obtained with VLT/UVES, for attempts to detect uranium. An additional set of 117 stars is scheduled to be observed in snapshot mode in Period 72 (April-September 2003).

\subsection{Carbon-enhanced metal-poor stars}

One of the early results from the HK survey was the finding that the frequency of carbon-enhanced stars increases dramatically at the lowest values of $[\mathrm{Fe} / \mathrm{H}]$, reaching $\sim 20-25 \%$ at $[\mathrm{Fe} / \mathrm{H}]<-2.5$ (Norris et al. 1997a; Rossi et al. 1999). This result has been confirmed by follow-up observations of HES candidates.

It turns out that nature presents us with a "zoo" of carbon-enhanced metal-poor (CEMP) stars, with vastly differing properties. Above all, the origin of carbon in this class of stars remains unclear, despite extensive investigations by means of highresolution spectroscopy (e. g., Norris et al. 1997a,b; Bonifacio et al. 1998; Hill et al. 2000; Aoki et al. 2002a; Norris et al. 2002; Lucatello et al. 2003). A larger sample of CEMP stars with abundance determinations, as well as long-term radial velocity monitoring, is needed to constrain the possible carbon enrichment scenarios realized in nature, and to separate known CEMP stars into the corresponding classes of objects.

We are currently increasing the number of CEMP stars in dedicated spectroscopic follow-up observations, mainly at the CTIO and KPNO $4 \mathrm{~m}$ telescopes. Confirmed CEMP stars from this and other efforts are studied at high resolution with Subaru/HDS and TNG/SARG. A program aiming at radial velocity monitoring of a large sample of CEMP stars has been proposed to be carried out with the AngloAustralian Telescope, and will hopefully be continued for many years with this, and other $4 \mathrm{~m}$-class telescopes in the future. 

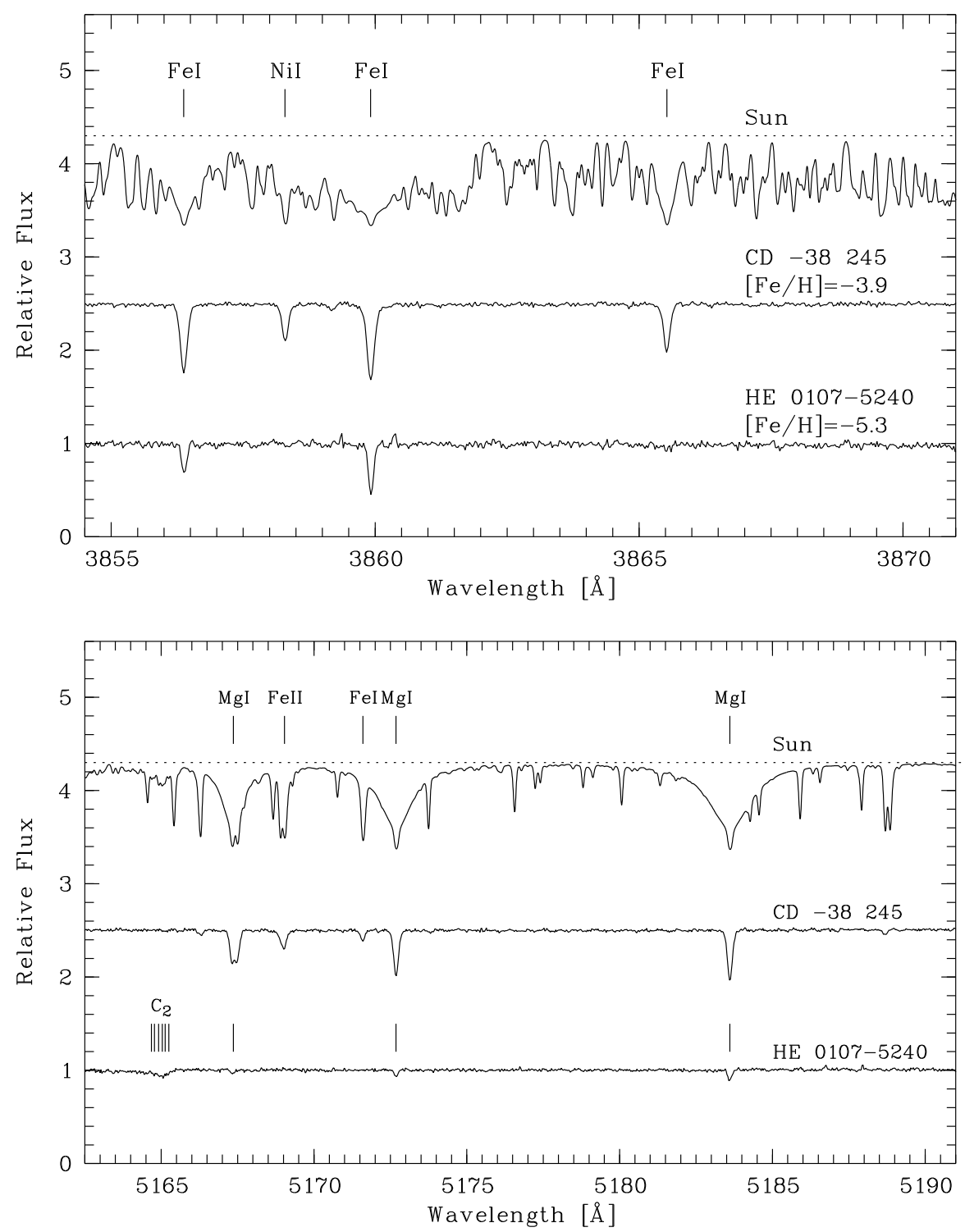

Figure 6: Spectrum of the Sun compared with the VLT/UVES spectrum of CD $-38^{\circ} 245$, the previously most metal-poor star, and with HE 0107-5240. The spectrum of CD $-38^{\circ} 245$ has been obtained with the same observational setup. The spectra are on the same scale and have been offset arbitrarily in the vertical direction. Note the very weak or absent Fe lines in the spectrum of HE 0107-5240, and the presence of the $\mathrm{C}_{2}$ band at $\sim 5165 \AA$. 


\subsection{HE 0107-5240}

Christlieb et al. (2002a) recently announced the discovery of HE0107-5240, a giant with $[\mathrm{Fe} / \mathrm{H}]=-5.3$. This star provides constraints on low-mass star formation in the early Galaxy, and insights into the properties of the first generation of stars and supernovae. The abundance pattern of HE0107-5240 is characterized by huge over-abundances of carbon and nitrogen with respect to iron $([\mathrm{C} / \mathrm{Fe}]=$ $+4.0 \mathrm{dex} ;[\mathrm{N} / \mathrm{Fe}]=+2.3 \mathrm{dex}$ ), while the abundances of the other five elements of with presently detected spectral lines exhibit a behavior similar to what is seen in other EMP stars.

Umeda \& Nomoto (2003) proposed a posteriori that the gas cloud from which HE 0107-5240 formed could have been enriched by a $25 M_{\odot}$ Population III star exploding as a supernova of low explosion energy $\left(E_{\text {exp }}=3 \cdot 10^{50} \mathrm{erg}\right)$. By assuming that the material produced during the $\mathrm{SN}$ event is homogeneously mixed over a wide range of the mass coordinate, and that a large fraction of the material falls back onto the compact remnant (the "mixing and fallback" mechanism), Umeda \& Nomoto are able to reproduce the abundance pattern of HE 0107-5240 remarkably well (see their Figure 1). In particular, very high $\mathrm{C} / \mathrm{Fe}$ and $\mathrm{N} / \mathrm{Fe}$ ratios can be produced in this scenario (with the CNO elements produced in late stages of the evolution of the SN progenitor), offering an interesting possibility for explaining why a large fraction of the extremely metal-poor stars exhibit high enhancements of carbon (for a similar work on the abundance patterns of CS 29498-043 and CS 22949-037 see Tsujimoto \& Shigeyama 2003).

The scenario of Umeda \& Nomoto is also very attractive in that it does not require the existence of a binary companion for the CEMP stars. In fact, there are at least three unevolved CEMP stars known that do not show any radial velocity variations larger than $0.4 \mathrm{~km} \mathrm{~s}^{-1}$ over a period of 8 years (Preston \& Sneden 2001), ruling out, at least for these three stars, the scenario in which a formerly more massive star went through its AGB phase and transfered dredged-up material onto the surface of the less massive companion, which we observe as a CEMP star today.

Umeda \& Nomoto predict an $\mathrm{O}$ abundance of $[\mathrm{O} / \mathrm{Fe}]=+2.8$ dex for HE $0107-$ 5240. A high-resolution, high $S / N$ spectrum of HE 0107-5240, covering the near UV region down to the atmospheric cutoff, has now been obtained with VLT/UVES. Spectrum synthesis calculations suggest that the detection of UV-OH lines is possible if $[\mathrm{O} / \mathrm{Fe}]>+0.3 \mathrm{dex}$. Therefore, the predictions of Umeda \& Nomoto can soon be tested.

\section{Acknowledgements}

I am grateful to D. Reimers (P. I. of the HES) and L. Wisotzki (HES project scientist) for making my work on the HES possible. It is a pleasure to acknowledge the contributions of my numerous collaborators to HES metal-poor star research over the last years. Here I would like to especially thank those who have participated in the huge effort of vetting HES candidates: Paul Barklem, Tim Beers, Mike Bessell, Innocenza Busa, Judy Cohen, Doug Duncan, Bengt Edvardson, Lisa Elliot, Birgit Fuhrmeister, Dionne James, Andreas Korn, Andy McWilliam, Solange Ramírez, Johannes Reetz, 
Jaehyon Rhee, Silvia Rossi, Sean Ryan, Steve Shectman, Ian Thompson, and FranzJosef Zickgraf. I thank Tim Beers for valuable comments, and proof-reading. This work is supported by Deutsche Forschungsgemeinschaft under grant Re 353/44-1, and was partly carried out during a Marie Curie Fellowship of the European Commission (contract number HPMF-CT-2002-01437) at Uppsala Astronomical Observatory, and a Linkage International Fellowship of the Australian Research Council (Project ID LX02114310) at Mt. Stromlo Observatory.

\section{References}

Aoki, W., Norris, J.E., Ryan, S.G., Beers, T.C., Ando, H. 2000, ApJ 536, L97

Aoki, W., Norris, J.E., Ryan, S.G., Beers, T.C., Ando, H. 2002a, ApJ 567, 1166

Aoki, W., Ryan, S.G., Norris, J.E., Beers, T.C., Ando, H., Tsangarides, S. 2002b, ApJ 580, 1149

Beers, T.C. 1999, in The Third Stromlo Symposium: The Galactic Halo, ed. B. Gibson, T. Axelrod, \& M. Putman, ASP Conf. Ser. 165, 202-212

Beers, T.C. 2000, in The First Stars, Proceedings of the second MPA/ESO workshop, ed. A. Weiss, T. Abel, \& V. Hill (Heidelberg: Springer), astro-ph/9911171

Beers, T.C., Preston, G.W., Shectman, S.A. 1985, AJ 90, 2089

Beers, T.C., Preston, G.W., Shectman, S.A. 1992, AJ 1987

Beers, T.C., Rossi, S., Norris, J.E., Ryan, S. G., Shefler, T. 1999, AJ 117, 981

Bonifacio, P., Molaro, P., Beers, T., Vladilo, G. 1998, A\&A 332, 672

Carretta, E., Gratton, R., Cohen, J., Beers, T., Christlieb, N. 2002, AJ 124, 481

Cayrel, R. 1996, A\&A Rev., 7, 217

Cayrel, R., Hill, V., Beers, T., Barbuy, B., Spite, M., Spite, F., Plez, B., Andersen, J., Bonifacio, P., Francois, P., Molaro, P., Nordström, B., Primas, F. 2001, Nature 409, 691

Christlieb, N., Beers, T. C. 2000, in Subaru HDS Workshop on stars and galaxies: Decipherment of cosmic history with spectroscopy, ed. M. Takada-Hidai \& H. Ando (Tokyo: National Astronomical Observatory), 255-273, astro-ph/0001378

Christlieb, N., Green, P., Wisotzki, L., Reimers, D. 2001a, A\&A 375, 366

Christlieb, N., Wisotzki, L., Reimers, D., Homeier, D., Koester, D., Heber, U. 2001b, A\&A 366,898

Christlieb, N., Bessell, M., Beers, T., Gustafsson, B., Korn, A., Barklem, P., Karlsson, T., Mizuno-Wiedner, M., Rossi, S. 2002a, Nature 419, 904

Christlieb, N., Wisotzki, L., Graßhoff, G. 2002b, A\&A 391, 397

Cohen, J., Christlieb, N., Beers, T., Gratton, R., Carretta, E. 2002, AJ 124, 26

Cohen, J., Christlieb, N., Qian, Y., Wasserburg, G. 2003, ApJ, in press, astro-ph/0301460

Cowan, J.J., McWilliam, A., Sneden, C., Burris, D.L. 1997, ApJ 480, 246

Cowan, J.J., Pfeiffer, B., Kratz, K.-L., Thielemann, F.-K., Sneden, C., Burles, S., Tytler, D., Beers, T. 1999, ApJ 521, 194

Fuhrmann, K. 1998, A\&A 338, 161

Goriely, S., Siess, L. 2001, A\&A 378, L25 
Hill, V., Barbuy, B., Spite, M., Spite, F., Plez, R.C.B., Beers, T., Nordström, B., Nissen, P. 2000, A\&A 353, 557

Hill, V., Plez, B., Cayrel, R., Nordström, T.B.B., Andersen, J., Spite, M., Spite, F., Barbuy, B., Bonifacio, P., Depagne, E., François, P., Primas, F. 2002, A\&A 387, 560

Karlsson, T., Gustafsson, B. 2001, A\&A 379, 461

Lucatello, S., Gratton, R., Carretta, E., Cohen, J., Christlieb, N., Beers, T., Ramírez, S. 2003, AJ 125,875

McWilliam, A., Preston, G., Sneden, C., Searle, L. 1995, AJ 109, 2757

Norris, J.E., Ryan, S.G., Beers, T.C. 1997a, ApJ 488, 350

Norris, J.E., Ryan, S.G., Beers, T.C. 1997b, ApJ 489, L169

Norris, J., Ryan, S., Beers, T. 2001, ApJ 561, 1034

Norris, J., Ryan, S., Beers, T., Aoki, W., Ando, H. 2002, ApJ 569, L107

Preston, G. 2000, PASP 112, 141

Preston, G., Sneden, C. 2001, AJ 122, 1545

Rhee, J. 2000, PhD thesis, Michigan State University

Rossi, S., Beers, T.C., Sneden, C. 1999, in The Third Stromlo Symposium: The Galactic Halo, ed. B. Gibson, T. Axelrod, \& M. Putman, ASP Conf. Ser. 165, 264-268)

Ryan, S., Norris, J., Beers, T. 1996, ApJ 471, 254

Ryan, S.G., Norris, J.E., Beers, T.C. 1999, ApJ 523, 654

Schatz, H., Toenjes, R., Pfeiffer, B., Beers, T., Cowan, J., Hill, V., Kratz, K. 2002, ApJ 579, 626

Shigeyama, T., Tsujimoto, T. 1998, ApJ 507, L135

Skrutskie, M., Schneider, S., Stiening, R., Strom, S., Weinberg, M., Beichman, C., Chester, T., Cutri, R., Lonsdale, C., Elias, J., Elston, R., Capps, R., Carpenter, J., Huchra, J., Liebert, J., Monet, D., Price, S., Seitzer, P. 1997, in The Impact of Large Scale Near-IR Sky Surveys, ed. F. Garzón, N. Epchtein, A. Omont, W. Burton, \& P. Persi (Dordrecht: Kluwer), 25-32

Sneden, C., McWilliam, A., Preston, G.W., Cowan, J.J., Burris, D.L., Amorsky, B.J. 1996, ApJ 467, 819

Sneden, C., Cowan, J., Ivans, I., Fuller, G., Burles, S., Beers, T., Lawler, J. 2000, ApJ 533, L139

Tsujimoto, T., Shigeyama, T., Yoshii, Y. 2000, ApJ 531, L33

Tsujimoto, T., Shigeyama, T. 2003, ApJ 584, L87

Umeda, H., Nomoto, K. 2003, Nature, in press, astro-ph/0301315

Van Eck, S., Goriely, S., Jorissen, A., Plez, B. 2001, Nature 412, 793

Wanajo, S., Itoh, N., Ishimaru, Y., Nozawa, S., Beers, T. 2002, ApJ 577, 853

Watson, F. 1998, AAO Newsletter, 11

Wisotzki, L., Christlieb, N., Bade, N., Beckmann, V., Köhler, T., Vanelle, C., Reimers, D. 2000, A\&A 358, 77 